\title{
The Pressurized Cylinder Problem for Nonlinear Viscoelastic Materials With a Strain Clock
}

\author{
Alan Wineman AND Je-Hong MiN \\ Department of Mechanical Engineering and Applied Mechanics, University of Michigan, Ann \\ Arbor, MI 48109
}

(Received 3 June 1996)

\begin{abstract}
A constitutive equation for nonlinear viscoelasticity is used to model the mechanical response of solid amorphous polymers such as polycarbonate. The nonlinearity arises from a reduced time, which causes stress relaxation to accelerate with increasing strain. This reduced time is referred to as a "strain clock". An important feature of the constitutive equation is that it accounts for yield under different strain and stress histories. This constitutive equation is used to study the problem of a hollow cylinder subjected to different pressure histories on its inner and outer surfaces. It is shown that if the strain clock depends only on the volumetric strain, then the governing equations admit a solution, which has a number of important consequences. First, the spatial distribution of displacements has the same form as for a linear elastic or linear viscoelastic material. The time evolution depends on the material properties. Second, if pressures are specified at the inner and outer surfaces, the resultant stress distribution is independent of material properties, and is the same as for linear elastic or linear viscoelastic response. Finally, it is found that the strain clock runs at the same rate for all radii. An experiment, based on these results, is suggested, which can be used to assess the assumption that the strain clock depends only on the volumetric strain.
\end{abstract}

\section{INTRODUCTION}

There has been a great deal of interest in a particular class of constitutive equations for the nonlinear viscoelastic response of amorphous polymers, such as polycarbonate, because of its ability to represent phenomena such yield. The dominant property of this class of constitutive equation is a material time scale or "clock" through which stress relaxation is accelerated with increasing strain. Substantial experimental and theoretical effort is being devoted to the evaluation of constitutive equations based on the clock concept. Shay and Caruthers [1] and Knauss and Emri [2,3] investigated forms of the constitutive equation in which stress relaxation is accelerated by volumetric strain. McKenna and Zapas [4] provided results that suggest shear deformations also accelerate stress relaxation.

There has been a parallel analytical effort in which the constitutive equation is used to study the interaction of the acceleration of stress relaxation with strain and the spatial 
variation of strain. This effort has been motivated by experiments involving nonhomogeneous deformations as well as structural applications in which polymeric materials may operate near yield. In most of the analytical work, it is assumed that there is geometric linearity and material nonlinearity. Moran and Knauss [5] studied the stresses near crack tips. Wineman and Waldron [6] considered circumferential shear of a cylinder. Wineman and Kolberg [7] and Kolberg [8] provided an extensive discussion of pure bending, while Min [9] extended the study to cantilever and simply supported beams under time dependent concentrated forces.

The present work considers a hollow cylinder, which is composed of a polymeric material having the constitutive properties discussed above. The cylinder is kept at a fixed length while different pressure histories are applied to the inner and outer surfaces. Analytical results are presented, which can be used to study the creep of polymeric pressurized containers, for accelerated stress relaxation implies accelerated creep. They also enable the stress and strain concentrations induced by the cylindrical cavity to be found for the nonlinear viscoelastic response. Yet a third application of the results is that they provide a theoretical basis for the interpretation of experiments, which use axially symmetric deformations of cylinders to study the behavior of polymers under biaxial and triaxial deformations.

The constitutive equation is presented in Section 2 and the problem is formulated in Section 3. The displacement satisfies a nonlinear partial differential-Volterra integral equation. It is shown that if the strain clock depends only on the volumetric strain or dilatation, then this equation admits an analytical solution. Owing to its complexity, the derivation of the governing equation, and its analytical solution, is carried out in the appendix. The analytical solution is explored in Section 4 and is shown to have several important consequences. First, at each time the displacement has the same dependence on the radius as does a linear elastic or linear viscoelastic material. The time evolution depend's on the material properties. Second, if pressures are specified at the inner and outer surfaces, the resultant stress distribution is independent of material properties, and is the same as for linear elastic or linear viscoelastic response. Third, it is found that the strain clock runs at the same rate for all radii. Results for a numerical example are presented in Section 5. Finally, an experiment is suggested, in Section 6, which can be used to assess the assumption that the strain clock depends only on the dilatation.

\section{NONLINEAR VISCOELASTIC CONSTITUTIVE EQUATION}

The displacement gradients are assumed to be sufficiently small that the linearized straindisplacement relations are valid. Let $\sigma_{i j}$ and $\varepsilon_{i j}$ denote components of the stress and strain tensor, respectively, with respect to a Cartesian coordinate system. The deviatoric part of the stress tensor is denoted by $\hat{\sigma}_{i j}=\sigma_{i j}-\frac{1}{3} \sigma_{k k} \delta_{i j}, \delta_{i j}$ is the Kronecker delta, and $\frac{1}{3} \sigma_{k k}$ denotes the hydrostatic part of the stress tensor. The deviatoric part of the strain tensor is denoted by $\hat{\varepsilon}_{i j}=\varepsilon_{i j}-\frac{1}{3} \varepsilon_{k k} \delta_{i j}$, and $\varepsilon_{k k}$ is the volumetric strain or dilatation. Let 
$\varepsilon_{k k}$ be denoted by $\theta$ and let $\hat{\varepsilon}=\left(\hat{\varepsilon}_{i j} \hat{\varepsilon}_{i j}\right)^{1 / 2}$, a deviatoric strain invariant.

It is assumed that the strains and stresses are zero for times less than zero, and that they may undergo a possible jump discontinuity at time zero. Then, the constitutive equation for isotropic linear viscoelastic response, in the regime of infinitesimal deformations, is

$$
\begin{aligned}
& \hat{\sigma}_{i j}=2 \int_{0-}^{t} \mu(t-s) \frac{\partial \hat{\varepsilon}_{i j}(s)}{\partial s} d s \\
& \sigma_{k k}=3 \int_{0-}^{t} K(t-s) \frac{\partial \theta(s)}{\partial s} d s
\end{aligned}
$$

where $\mu(t)$ is the shear relaxation function and $K(t)$ is the bulk relaxation function. As in $[2,3]$, the constitutive equation for isotropic nonlinear viscoelastic response is

$$
\begin{aligned}
& \hat{\sigma}_{i j}=2 \int_{0-}^{t} \mu[\xi(t)-\xi(s)] \frac{\partial \hat{\varepsilon}_{i j}(s)}{\partial s} d s, \\
& \sigma_{k k}=3 \int_{0-}^{t} K[\xi(t)-\xi(s)] \frac{\partial \theta(s)}{\partial s} d s,
\end{aligned}
$$

where $\xi(t)$ is defined in terms of the strain history by

$$
\xi(t)=\int_{0}^{t} \frac{d x}{\phi(\theta(x), \hat{\varepsilon}(x))} .
$$

The dependence of $\xi(t)$ on the dilatation $\theta$ and the deviatoric strain invariant $\hat{\varepsilon}$ causes the constitutive equation to be nonlinear in the strains.

The lower limit in the integrals allows for a possible jump discontinuity in strain at time zero. In this case, the notation, illustrated for equation (3), represents the following,

$$
\int_{0-}^{t} \mu[\xi(t)-\xi(s)] \frac{\partial \hat{\varepsilon}_{i j}(s)}{\partial s} d s=\mu[\xi(t)] \hat{\varepsilon}_{i j}(0)+\int_{0}^{t} \mu[\xi(t)-\xi(s)] \frac{\partial \hat{\varepsilon}_{i j}(s)}{\partial s} d s
$$

and the 0 on the right hand side represents $0+$.

Equation (5) defines a new variable $\xi(t)$ called a material time (also referred to as pseudo, intrinsic, or reduced time) and relates it to the physical or laboratory time $t$. $\xi(t)$ is often described as the time determined by a material "clock," in this case a "strain clock." The material clock can run at different speeds relative to the physical or laboratory clock, depending on the strain history.

The integrand $\phi$ has properties similar to those of the time-temperature shift function and is a monotonically decreasing function of $\theta$ and $\hat{\varepsilon}$. Thus deformation histories for which $\theta$ and $\hat{\varepsilon}$ increase with time cause $\xi(t)$ to increase faster than the physical time $t$. This results in an acceleration of stress relaxation. Wineman and Waldron showed that if the strain history is such that $\phi$ becomes sufficiently small, then the constitutive equation simulates yield in polymers. The form for $\phi$ used by $[2,3]$ and $[6]$ is

$$
\log \phi=\frac{b}{2.303}\left(\frac{1}{f}-\frac{1}{f_{0}}\right),
$$


where $b$ is a material property and is a constant. Knauss and Emri interpreted $f$ as the fractional free volume and related it to the dilatation, that is, $f=f(\theta)$. Then, $f_{0}$ denotes the value of the fractional free volume in the reference state. For now, it is assumed that $f$ depends on both the dilatation and deviatoric strain, that is, $f=f(\theta, \hat{\varepsilon})$.

\section{PROBLEM FORMULATION}

Consider a hollow cylinder, with inner radius $r_{i}$ and outer radius $r_{0}$, made of an isotropic nonlinear viscoelastic material whose constitutive equation is described in Section 2. The cylinder is in an undeformed, stress-free configuration for times less than zero. Uniform pressures are applied to its inner and outer surfaces for times $t \geq 0$ and there are no body forces. The deformation is assumed to be axially symmetric and quasi-static.

Let the 1, 2, and 3 directions denote the $r, \theta$, and $z$ directions, respectively, of a cylindrical coordinate system and let $u_{i}$ represent the displacement components. It is assumed that there is only a radial displacement, thus

$$
u_{1}=u(r, t), \quad u_{2}=u_{3}=0
$$

At each instant, there are no shear strains and the normal strain components are given by

$$
\varepsilon_{11}(r, t)=\frac{\partial u(r, t)}{\partial r}, \quad \varepsilon_{22}(r, t)=\frac{u(r, t)}{r}, \quad \varepsilon_{33}=0 .
$$

From equations (3), (4), and (9), the stress components are given by

$$
\begin{aligned}
\sigma_{11}(r, t)= & 2 \int_{0-}^{t} \mu[\xi(r, t)-\xi(r, s)] \frac{\partial \varepsilon_{11}(r, s)}{\partial s} d s \\
& +\int_{0-}^{t}\left[K[\xi(r, t)-\xi(r, s)]-\frac{2}{3} \mu[\xi(r, t)-\xi(r, s)]\right] \frac{\partial \theta(r, s)}{\partial s} d s, \\
\sigma_{22}(r, t)= & 2 \int_{0-}^{t} \mu[\xi(r, t)-\xi(r, s)] \frac{\partial \varepsilon_{22}(r, s)}{\partial s} d s \\
& +\int_{0-}^{t}\left[K[\xi(r, t)-\xi(r, s)]-\frac{2}{3} \mu[\xi(r, t)-\xi(r, s)]\right] \frac{\partial \theta(r, s)}{\partial s} d s, \\
\sigma_{33}(r, t)= & \int_{0-}^{t}\left[K[\xi(r, t)-\xi(r, s)]-\frac{2}{3} \mu[\xi(r, t)-\xi(r, s)]\right] \frac{\partial \theta(r, s)}{\partial s} d s,
\end{aligned}
$$

in which $\theta(r, s)$ denotes the dilatation, given by

$$
\theta(r, s)=\varepsilon_{11}(r, s)+\varepsilon_{22}(r, s)+\varepsilon_{33}(r, s)=\frac{\partial u(r, s)}{\partial r}+\frac{u(r, s)}{r}
$$

and, by (5),

$$
\xi(r, t)=\int_{0}^{t} \frac{d x}{\phi(\theta(r, x), \hat{\varepsilon}(r, x))} .
$$


Because the deformation is assumed to be quasistatic at each instant, the spatial distribution of stresses must satisfy the equilibrium equation

$$
\frac{\partial \sigma_{11}(r, t)}{\partial r}+\frac{\sigma_{11}(r, t)-\sigma_{22}(r, t)}{r}=0 .
$$

Let the pressures over the inner and outer surfaces of the cylinder be denoted by $P_{i}(t)$ and $P_{0}(t)$, respectively. Then, the boundary conditions on the cylinder are

$$
\sigma_{11}\left(r_{i}, t\right)=-P_{i}(t), \quad \sigma_{11}\left(r_{0}, t\right)=-P_{0}(t)
$$

Substitution from equations (9) through (14) into equilibrium equation (15) results in a nonlinear partial differential - Volterra integral equation for $u(r, t)$.

\section{A SOLUTION}

The special case is now considered in which $f=f(\theta)$ in equation (7), that is, the strain clock depends only on the dilatation. Equation (14) then reduces to

$$
\xi(r, t)=\int_{0}^{t} \frac{d x}{\phi(\theta(r, x))} .
$$

It is shown in the appendix that a sufficient condition for the system consisting of equations (9) through (11), (15) and (17) to be satisfied, is

$$
\frac{\partial}{\partial r}\left(\frac{\partial u(r, t)}{\partial r}\right)+\frac{1}{r} \frac{\partial u(r, t)}{\partial r}-\frac{1}{r^{2}} u(r, t)=0
$$

for $r_{i} \leq r \leq r_{0}$ and $t \geq 0$. Equation (18) has the general solution

$$
u(r, t)=c_{1}(t) r+c_{2}(t) \frac{1}{r}
$$

where $c_{1}(t)$ and $c_{2}(t)$ are arbitrary functions of time and are determined by the boundary conditions. By equations (9) and (19), the strains are

$$
\begin{aligned}
& \varepsilon_{11}(r, t)=\frac{\partial u(r, t)}{\partial r}=c_{1}(t)-\frac{c_{2}(t)}{r^{2}} \\
& \varepsilon_{22}(r, t)=\frac{u(r, t)}{r}=c_{1}(t)+\frac{c_{2}(t)}{r}
\end{aligned}
$$

and the dilatation in equation (13) is

$$
\theta(r, t)=\varepsilon_{11}(r, t)+\varepsilon_{22}(r, t)=2 c_{1}(t) .
$$


According to equation (21), the dilatation $\theta(r, t)$ is independent of the radius and varies only with $t$. Thus, by equation (17), the reduced time $\xi(r, t)$ is also independent of the radius, that is,

$$
\xi(r, t)=\int_{0}^{t} \frac{d x}{\phi\left(2 c_{1}(x)\right)}=\xi(t) .
$$

Let equations (20) and (21) be substituted into equations (10) and (11), and use be made of equation (22). The stresses then become expressed as follows in terms of $c_{1}(t)$, $c_{2}(t)$ and $r$,

$$
\begin{aligned}
\sigma_{11}(r, t)= & 2 \int_{0-}^{t} \mu[\xi(t)-\xi(s)] \frac{\partial}{\partial s}\left[c_{1}(s)-\frac{c_{2}(s)}{r^{2}}\right] d s \\
& +\int_{0-}^{t}\left[K[\xi(t)-\xi(s)]-\frac{2}{3} \mu[\xi(t)-\xi(s)]\right] \frac{\partial\left(2 c_{1}(s)\right)}{\partial s} d s \\
= & {\left[\int_{0-}^{t}\left[K[\xi(t)-\xi(s)]+\frac{1}{3} \mu[\xi(t)-\xi(s)]\right] \frac{\partial\left(2 c_{1}(s)\right)}{\partial s} d s\right] } \\
& -\left[2 \int_{0-}^{t} \mu[\xi(t)-\xi(s)] \frac{\partial\left(c_{2}(s)\right)}{\partial s} d s\right] \frac{1}{r^{2}}, \\
\sigma_{22}(r, t)= & 2 \int_{0-}^{t} \mu[\xi(t)-\xi(s)] \frac{\partial}{\partial s}\left[c_{1}(s)+\frac{c_{2}(s)}{r^{2}}\right] d s \\
& +\int_{0-}^{t}\left[K[\xi(t)-\xi(s)]-\frac{2}{3} \mu[\xi(t)-\xi(s)]\right] \frac{\partial\left(2 c_{1}(s)\right)}{\partial s} d s \\
= & {\left[\int_{0-}^{t}\left[K[\xi(t)-\xi(s)]+\frac{1}{3} \mu[\xi(t)-\xi(s)]\right] \frac{\partial\left(2 c_{1}(s)\right)}{\partial s} d s\right] } \\
& +\left[2 \int_{0-}^{t} \mu[\xi(t)-\xi(s)] \frac{\partial\left(c_{2}(s)\right)}{\partial s} d s\right] \frac{1}{r^{2}}, \\
\sigma_{33}(t)= & {\left[\int_{0-}^{t}\left[K[\xi(t)-\xi(s)]-\frac{2}{3} \mu[\xi(t)-\xi(s)]\right] \frac{\partial\left(2 c_{1}(s)\right)}{\partial s} d s\right] . }
\end{aligned}
$$

When the boundary conditions in equation (16) are applied, explicit expressions for the integrals in equations (23) and (24) are found, and the stresses become

$$
\begin{aligned}
& \sigma_{11}(r, t)=\frac{-P_{0}(t)\left[\left(\frac{r_{0}}{r_{i}}\right)^{2}-\left(\frac{r_{0}}{r}\right)^{2}\right]-P_{i}(t)\left[\left(\frac{r_{0}}{r}\right)^{2}-1\right]}{\left(\frac{r_{0}}{r_{i}}\right)^{2}-1}, \\
& \sigma_{22}(r, t)=\frac{-P_{0}(t)\left[\left(\frac{r_{0}}{r_{i}}\right)^{2}+\left(\frac{r_{0}}{r}\right)^{2}\right]+P_{i}(t)\left[1+\left(\frac{r_{0}}{r}\right)^{2}\right]}{\left(\frac{r_{0}}{r_{i}}\right)^{2}-1} .
\end{aligned}
$$

Note that these expressions for the stresses are independent of the properties of the class of materials under consideration, namely nonlinearly viscoelastic materials having a dilatation dependent clock. Thus, when the pressure histories $P_{i}(t)$ and $P_{0}(t)$ are specified, 
the histories of the radial and circumferential stresses are known at each material element. If step pressure histories are applied, each material element experiences a step stress history, in which the magnitude of the step in the stress varies with radius. If each pressure increases at a constant rate, then each material element experiences a constant stress rate history, where stress rates vary with radius. It is also interesting to note that these stresses are the same as would occur in a cylinder composed of a linear elastic or a linear viscoelastic material.

Although the stresses do not depend on material properties, the strains, dilatation and radial displacement do. Functions $c_{1}(t)$ and $c_{2}(t)$ in equation (19) for the radial displacement appear in the integrals in equations (23) and (24). These integrals, found by applying the boundary condition in equation (16), are

$$
\begin{gathered}
\int_{0-}^{t}\left[K[\xi(t)-\xi(s)]+\frac{1}{3} \mu[\xi(t)-\xi(s)]\right] \frac{\partial\left[2 c_{1}(s)\right]}{\partial s} d s=\frac{P_{i}(t)-P_{0}(t)\left(\frac{r_{0}}{r_{i}}\right)^{2}}{\left[\left(\frac{r_{0}}{r_{i}}\right)^{2}-1\right]} \\
2 \int_{0-}^{t}[\mu[\xi(t)-\xi(s)]] \frac{\partial\left[c_{2}(s) / r_{i}^{2}\right]}{\partial s} d s=\frac{\left(P_{i}(t)-P_{0}(t)\right)\left(\frac{r_{0}}{r_{i}}\right)^{2}}{\left[\left(\frac{r_{0}}{r_{i}}\right)^{2}-1\right]}
\end{gathered}
$$

This is a system of nonlinear Volterra integral equations for $c_{1}(t)$ and $c_{2}(t)$. Numerical methods for solving such equations have been discussed by [6] and are omitted here for the purpose of brevity. Once $c_{1}(t)$ and $c_{2}(t)$ have been found, the strains, displacement, and dilatation are easily obtained from equations (19), (20), and (21).

\section{NUMERICAL RESULTS}

For the purpose of a numerical example, it is necessary to specify forms for the material properties. The shear relaxation function $\mu(t)$ and the bulk relaxation function $K(t)$ are assumed to have the forms

$$
\begin{gathered}
\mu(t)=\mu_{0}+\left(\mu_{\infty}-\mu_{0}\right)\left(1-e^{-t / \tau_{\mu}}\right), \\
K(t)=K_{0}+\left(K_{\infty}-K_{0}\right)\left(1-e^{-t / \tau_{K}}\right),
\end{gathered}
$$

Function $\phi(f)$ is defined in equation (7). Knauss and Emri [2,3] assumed that $f(\theta)$ is linear in $\theta$. Here it is assumed that

$$
f(\theta)=f_{0}+c|\theta|
$$

where $f_{0}$ denotes the fractional free volume in the reference state and $c$ is a material constant. With this formulation, both volume increase and decrease accelerate stress 
relaxation. Yield is observed both in extension, where there is volume increase, and in compression, where there is volume decrease. This suggests, for present purposes, that $f(\theta)$ be assumed to depend only on the magnitude of the dilatation. Values for the parameters in these properties were taken from [2, 3]: $\mu_{0}=635.0 \mathrm{MPa}, \mu_{\infty}=0.2 \mathrm{MPa}$, $K_{0}=3350.0 \mathrm{MPa}, K_{\infty}=1340.0 \mathrm{MPa}, \tau_{K} / \tau_{\mu}=20$. The parameters in equations (7) and (32) are $b=0.16, c=1.00, f_{0}=0.01$.

The following nondimensional variables are introduced:

$$
\begin{gathered}
\bar{t}=t / \tau_{\mu}, \quad \bar{s}=s / \tau_{\mu}, \quad \bar{\xi}(\bar{t})=\xi(t) / \tau_{\mu}, \\
\bar{\mu}(\bar{t})=\mu(\bar{t}) / \mu_{0}, \quad \bar{K}(\bar{t})=K(\bar{t}) / \mu_{0}, \\
\bar{r}=r / r_{i} \quad \bar{P}_{i}(\bar{t})=P_{i}(\bar{t}) / \mu_{0}, \quad \bar{u}=u / r_{i} .
\end{gathered}
$$

For the purpose of brevity, only the case of internal pressure is considered, that is, $P_{0}(t)=$ 0 . Numerical results are presented for $r_{0} / r_{i}=2$, with the constant pressure rate history, $\bar{P}_{i}(\bar{t})=\left(345 / \mu_{0}\right) \bar{t}$, and for $r_{0} / r_{i}=5$, with the constant pressure rate history, $\bar{P}_{i}(\bar{t})=$ $\left(840 / \mu_{0}\right) \bar{t}$. These pressure histories were chosen so that the maximum strain would meet the condition that $\varepsilon_{\max } \approx 0.08$ at the final simulation time $\bar{t}=0.1$. Each figure shows results for the linear viscoelastic case (LVE, $c=0.0$ in equation (7)) and the nonlinear viscoelastic case (NLVE, $c=1.00$ in equation (7)).

In view of equation (21), the dilatation is obtained by solving equations (22) and (28). The dilatation histories for $r_{0} / r_{i}=2$ and $r_{0} / r_{i}=5$ are shown in Figures 1 and 2, respectively. These figures show that the dilatation is positive for all times and increases monotonically for both the LVE and NLVE cases. The histories for the LVE and NLVE cases are very close for times $\bar{t}$ when $\theta<0.001$. For later times, the dilatation has become large enough to influence the material time, stress relaxation and creep. The dilatation for the NLVE case then begins to increase faster than that for the LVE case. Because of the above-mentioned constraint on the pressure histories, the dilatation for $r_{0} / r_{i}=2$ becomes much larger than that for $r_{0} / r_{i}=5$. As a consequence, when $r_{0} / r_{i}=2$ the material clock runs faster and there is greater deviation between the LVE and NLVE cases.

Figure 3 shows the radial strain distribution at several times for the cylinder with $r_{0} / r_{i}=2$. The difference between the LVE and NLVE displacements increases with time due to the acceleration of stress relaxation. The difference is very small at first, until the dilatation becomes large enough so that stress relaxation is significantly affected. The difference then grows very rapidly between $\bar{t}=0.06$ and $\bar{t}=0.10$. In particular, at $\bar{t}=0.10$, the displacement at the inner surface $\bar{r}=1$ for NLVE is almost two times larger than the displacement for LVE. Figure 4 shows the radial displacement distributions for $r_{0} / r_{i}=5$. The difference between the LVE and NLVE displacements increases in a manner similar to that when $r_{0} / r_{i}=2$, but much more slowly. The reason for this was discussed in conjunction with the dilatation. The radial strain distribution for $r_{0} / r_{i}=5$ differs significantly from that when $r_{0} / r_{i}=2$. Its magnitude decreases rapidly with radius to values that are much smaller than for $r_{0} / r_{i}=2$.

The radial displacement distributions for several times are shown in Figure 5 for $r_{0} / r_{i}=2$ and in Figure 6 for $r_{0} / r_{i}=5$. The features are similar to those for the radial 


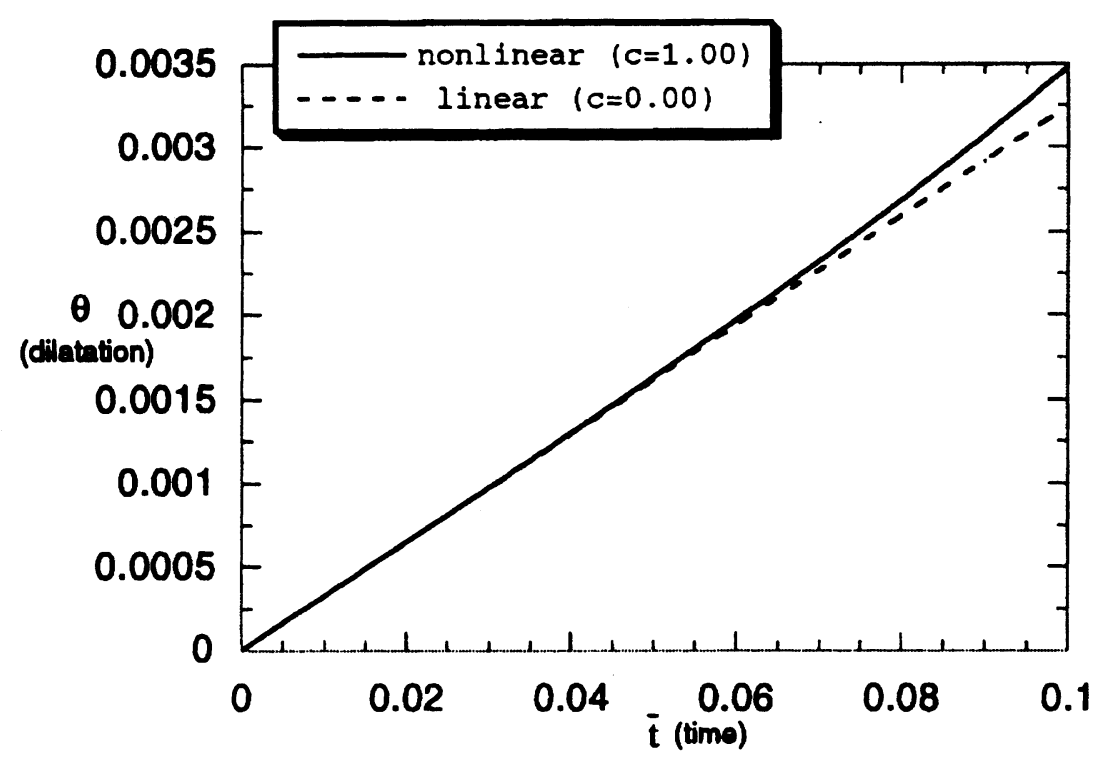

Fig. 1. Dilatation versus time, $r_{0} / r_{i}=2$.

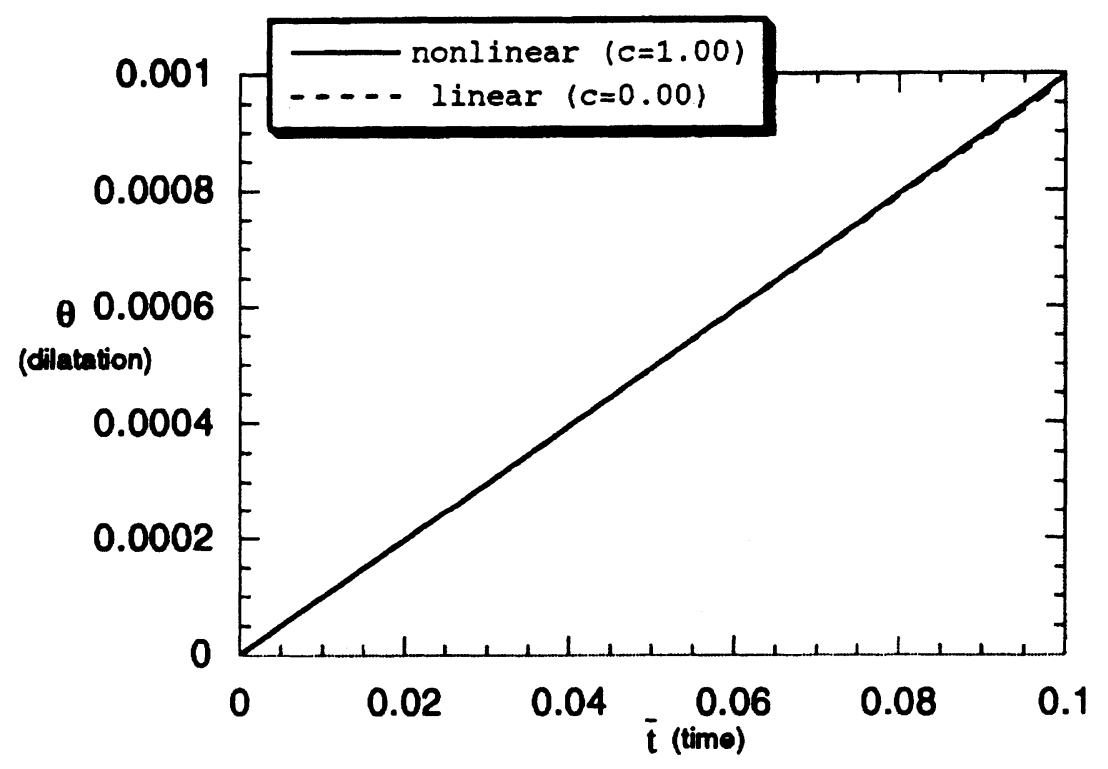

Fig. 2. Dilatation versus time, $r_{0} / r_{i}=5$. 


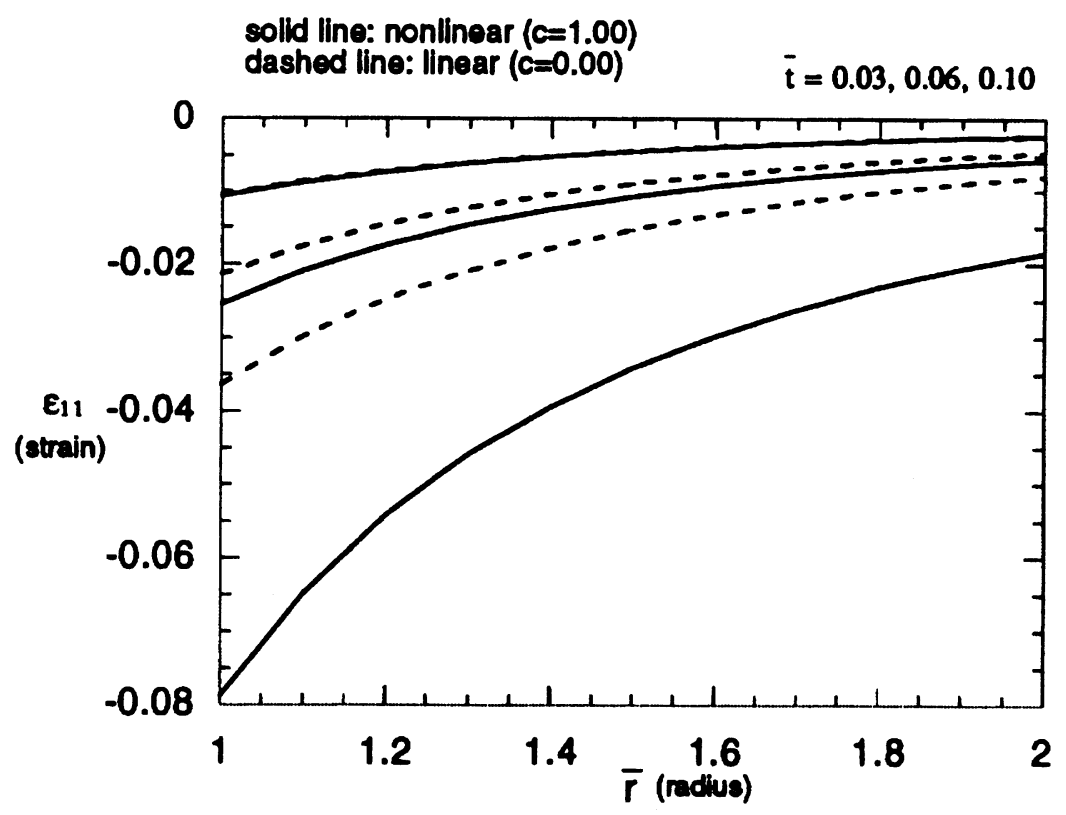

Fig. 3. Radial Strain versus radius for several times, $r_{0} / r_{i}=2$.

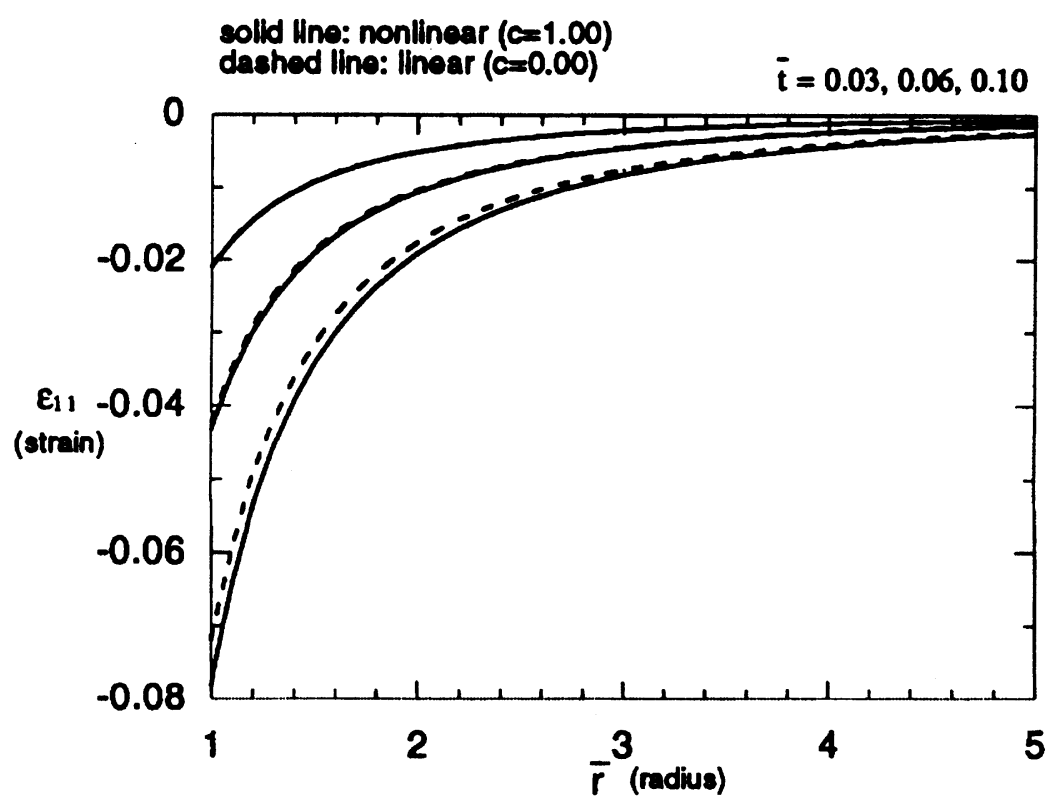

Fig. 4. Radial Strain versus radius for several times, $r_{0} / r_{i}=5$. 


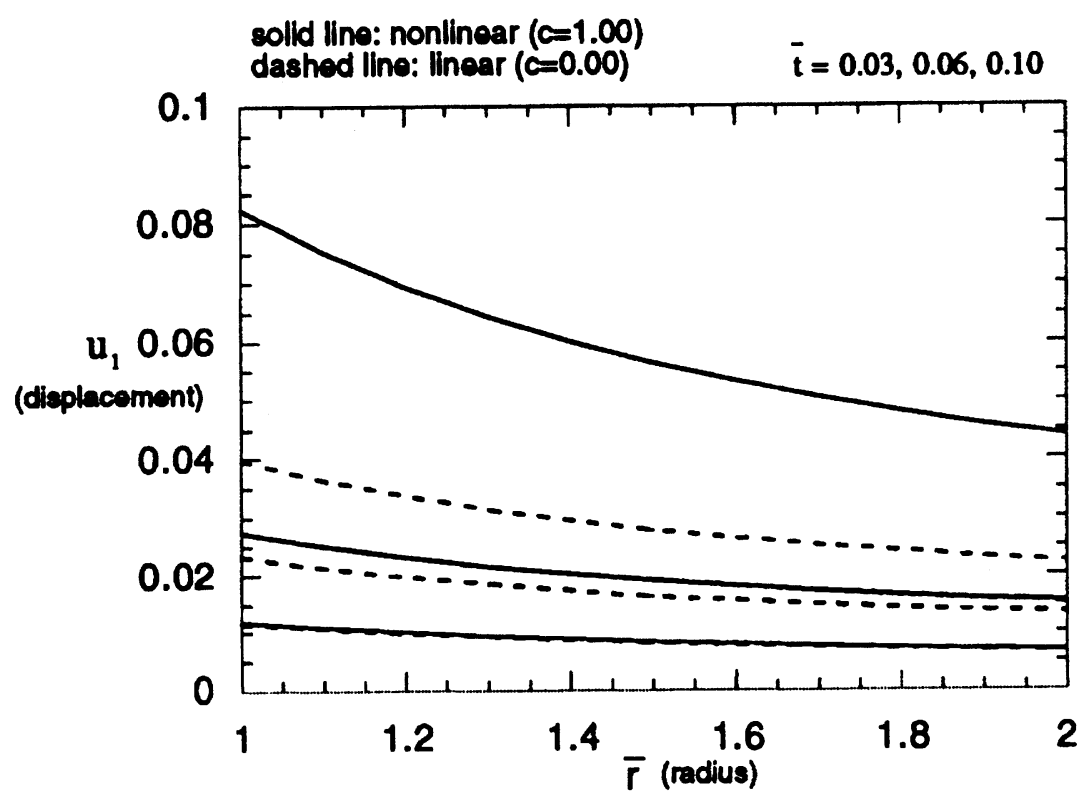

Fig. 5. Displacement versus radius for several times, $r_{0} / r_{i}=2$.

strain distribution shown in Figures 3 and 4. The LVE and NLVE radial displacement histories at various radii are shown in Figure 7, for $r_{0} / r_{i}=2$. The displacements increase monotonically, with that at the inner surface increasing more rapidly than that at the outer surface. The LVE and NLVE histories coincide for times $\bar{t}<0.02$. Since the dilatation and material time are independent of radius, the influence of the accelerated stress relaxation begins to show at all radii simultaneously. Thus, the NLVE histories deviate simultaneously from the LVE histories. This deviation increases at an ever increasing rate due to the growth in dilatation and the consequent increase in material time. Figure 8 shows LVE and NLVE displacement histories for $r_{0} / r_{i}=5$. Their behavior is similar to that when $r_{0} / r_{i}=2$.

There are several important comments regarding the results presented here. First, because of the assumption that $f$ depends only on the magnitude of the dilatation, the governing equations are still satisfied by setting $P_{i}(t) \rightarrow-P_{i}(t), P_{0}(t) \rightarrow-P_{0}(t), c_{\alpha}(t) \rightarrow$ $-c_{\alpha}(t), u(r, t) \rightarrow-u(r, t)$, and $\theta(t) \rightarrow-\theta(t)$. Thus the numerical results apply equally well to the growth of a cavity in a cylinder under radial tension. Second, the numerical results show that the displacements for LVE and NLVE are very close and grow very slowly for some initial time interval when the dilatation is sufficiently small. Then, when the dilatation is sufficiently large that NLVE effects become significant, the displacement can grow very rapidly. In other terms, one consequence of the acceleration of stress relaxation is the onset of rapid changes after some initial time interval of slow steady response. Third, the results of Section 4 are based on the assumption in equation (7) that the function $f$ depends only on the dilatation. If function $f$ should also depend on the 


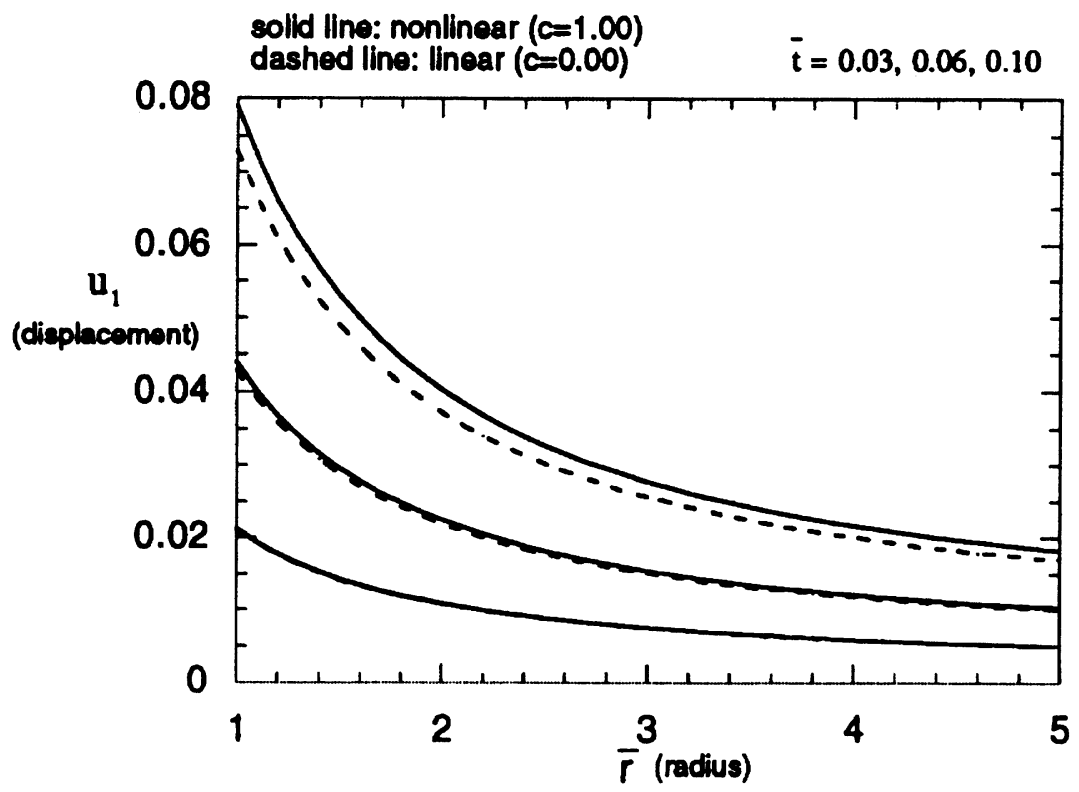

Fig. 6. Displacement versus radius for several times, $r_{0} / r_{i}=5$.

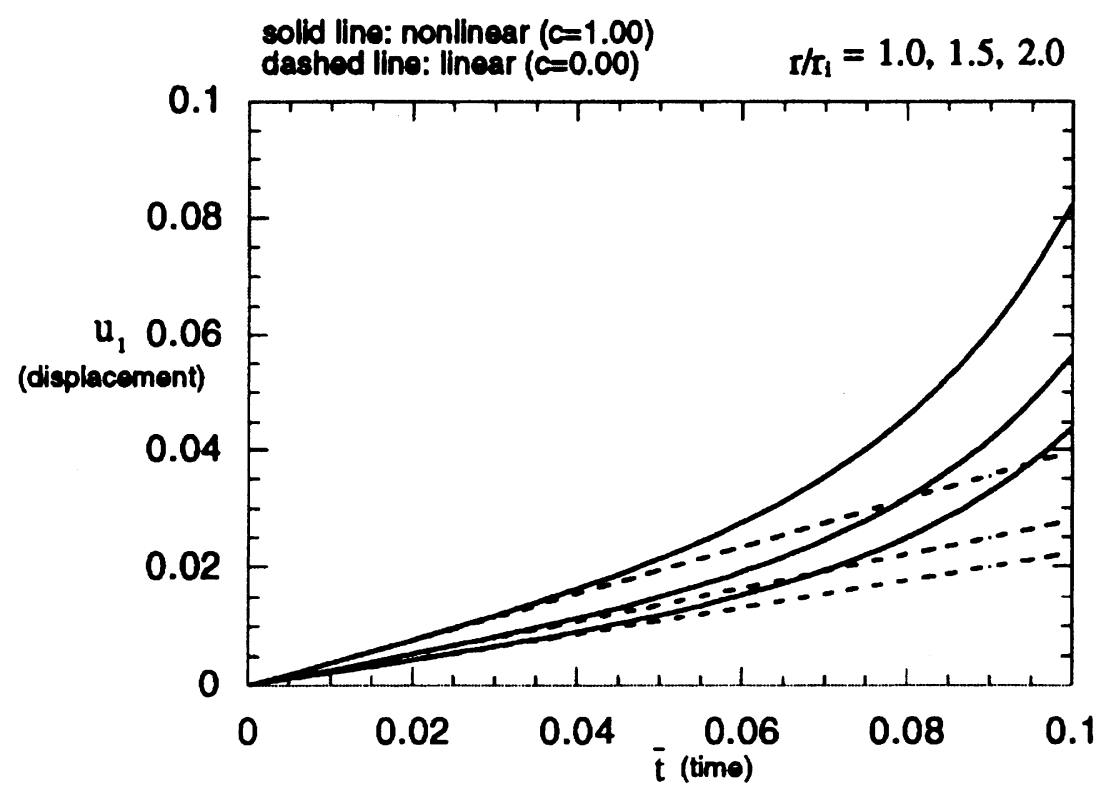

Fig. 7. Displacement versus time for several radii, $r_{0} / r_{i}=2$. 


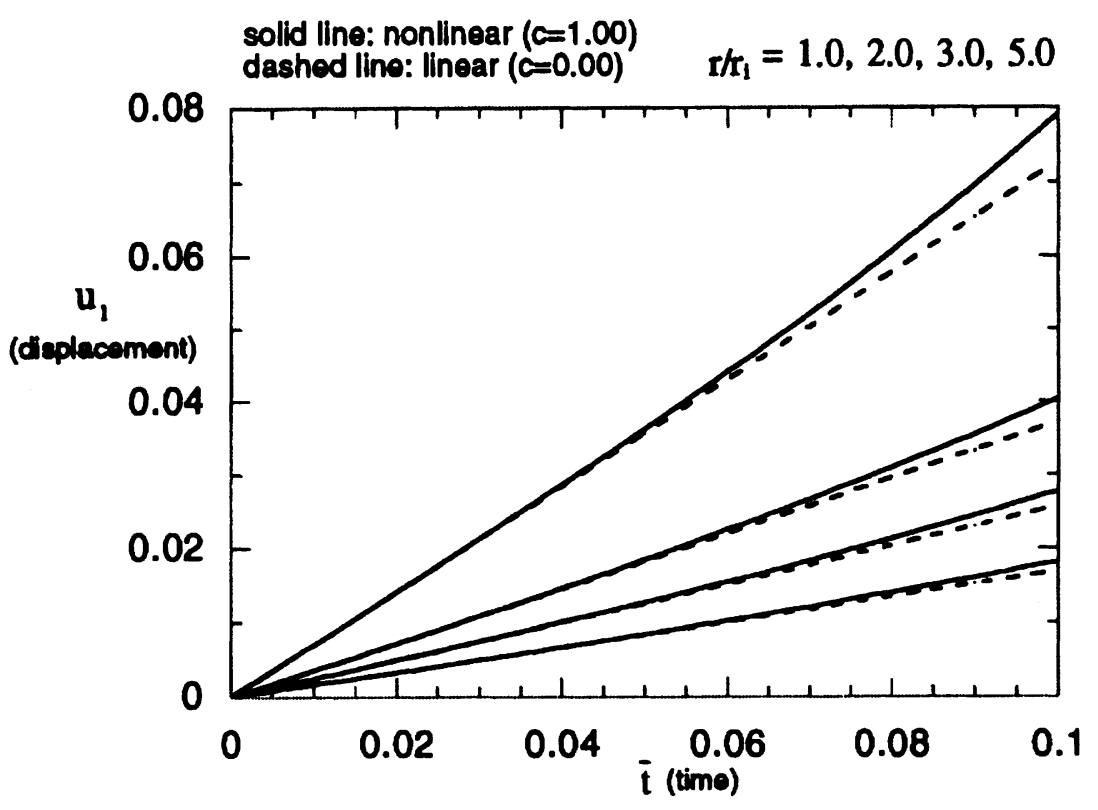

Fig. 8. Displacement versus time for several radii, $r_{0} / r_{i}=5$.

deviatoric components, then the displacement would no longer satisfy equation (18) and these results would no longer be valid. Thus it is of interest to develop an experiment based on these results that can test the validity of the assumption that $f$ depends only on the dilatation. This is done in the next section.

\section{IMPLICATIONS OF RESULTS FOR MODEL VERIFICATION}

The assumption that $f=f(\theta)$ in equation (7) leads to the specific expression for the radial displacement in equation (19). One consequence of this result is that the dilatation in equation (21) and the material time in (22) are independent of the radius. Another is that the time dependent functions in equation (19) are related to the material properties and the pressures by equations (28) and (29). These results are now used to suggest an experiment for testing the validity of the underlying assumption.

It is assumed that the shear relaxation function $\mu(t)$ and the bulk relaxation function $K(t)$ are known from measurements in the LVE range. It is further assumed that the displacement and pressure at the inner and outer surfaces can be measured. Let $u_{i}(t)=u\left(r_{i}, t\right)$ and $u_{0}(t)=u\left(r_{0}, t\right)$. The functions $c_{1}(t)$ and $c_{2}(t)$ in equation (19) can be expressed in terms of the measured displacements at the inner and outer surfaces by the 
relations

$$
\begin{aligned}
& c_{1}(t)=\frac{\left(\frac{r_{0}}{r_{i}}\right)^{2}\left(\frac{u_{0}(t)}{r_{0}}\right)-\frac{u_{i}(t)}{r_{i}}}{\left(\frac{r_{0}}{r_{i}}\right)^{2}-1}, \\
& \frac{c_{2}(t)}{r_{i}^{2}}=-\frac{\left(\frac{r_{0}}{r_{i}}\right)^{2}\left(\frac{u_{0}(t)}{r_{0}}-\frac{u_{i}(t)}{r_{i}}\right)}{\left(\frac{r_{0}}{r_{i}}\right)^{2}-1} .
\end{aligned}
$$

Note that, by equations (21) and (34), the dilatation is also expressed in terms of the measured displacements at the inner and outer surfaces.

Let the pressure histories $P_{i}(t)$ and $P_{0}(t)$ be such that they produce a step dilatation history, $\theta(t)=2 c_{1}(t)=\theta_{0}, t \geq 0$, and denote them by $\hat{P}_{i}(t)$ and $\hat{P}_{0}(t)$. Then, by equation (22),

$$
\xi(t)=\int_{0}^{t} \frac{d x}{\phi\left(\theta_{0}\right)}=\frac{t}{\phi\left(\theta_{0}\right)},
$$

that is, the material time reduces to a uniform scaling of the laboratory time. Equations (28) and (29) become

$$
\begin{gathered}
M\left[\frac{t}{\phi\left(\theta_{0}\right)}\right] \theta_{0}=\frac{\hat{P}_{i}(t)-\hat{P}_{0}(t)\left(\frac{r_{0}}{r_{i}}\right)^{2}}{\left[\left(\frac{r_{0}}{r_{i}}\right)^{2}-1\right]} \\
2 \int_{0-}^{t}\left[\mu\left[\frac{t-s}{\phi\left(\theta_{0}\right)}\right]\right] \frac{\partial\left[c_{2}(s) / r_{i}^{2}\right]}{\partial s} d s=\frac{\left(\hat{P}_{i}(t)-\hat{P}_{0}(t)\right)\left(\frac{r_{0}}{r_{i}}\right)^{2}}{\left[\left(\frac{r_{0}}{r_{i}}\right)^{2}-1\right]}
\end{gathered}
$$

in which $M(t)=K(t)+\frac{1}{3} \mu(t)$.

Now suppose that $\theta_{0} M(t)$ and the expression on the right hand side of equation (37) is each plotted versus $\log t$. According to equation (37), these should be related by a rigid shift or translation along the $\log t$ axis. This provides one test of the validity of the underlying assumption.

If the measured results satisfy equation (37), the amount of the shift gives $\phi\left(\theta_{0}\right)$. Next, substitute equation (35) into equation (38). The result is

$$
2 \int_{0-}^{t} \mu\left[\frac{t-s}{\phi\left(\theta_{0}\right)}\right] \frac{\partial}{\partial s}\left(\frac{u_{0}(s)}{r_{0}}-\frac{u_{i}(s)}{r_{i}}\right) d s=\left(\hat{P}_{i}(t)-\hat{P}_{0}(t)\right) .
$$

This equation can be inverted to give

$$
\int_{0-}^{t} \mu^{-1}\left[\frac{t-s}{\phi\left(\theta_{0}\right)}\right] \frac{\partial}{\partial s}\left(\hat{P}_{i}(s)-\hat{P}_{0}(s)\right) d s=\frac{1}{2}\left(\frac{u_{0}(t)}{r_{0}}-\frac{u_{i}(t)}{r_{i}}\right),
$$

where $\mu^{-1}(t)$ is the shear creep compliance. A second test of the underlying assumption is that the measured displacements and pressures must satisfy equation (39) or (40). 


\section{CONCLUDING REMARKS}

A number of significant results have been obtained for the class of NLVE materials with dilatation clock considered here. The first is that the dilatation clock is independent of the radius. This led to the second result, that the radial and circumferential stresses are independent of the material properties when pressures are specified. The third is that these stresses are the same as in cylinders composed of linear elastic or linear viscoelastic materials. Thus, even though stress relaxation occurs faster with increasing dilatation, this has no effect on the stresses when pressures are specified on the inner and outer surfaces of the cylinder. In particular, there is no influence on the stress concentration induced by a cylindrical cavity. However, the creep response is significantly affected. The numerical example shows that when NLVE effects become significant , the displacement and hence the strains can grow very rapidly after some initial period of slow steady increase.

This last comment indicates the need for two important extensions of this study. The first is the inclusion of the inertia of the material, which has been neglected here. The second extension arises from consideration of the magnitude of the strains. As can be seen from equation (9) and the numerical results for the radial displacement, the strains in the NLVE case can rapidly exceed the range of validity of linearized strain measures. The present constitutive equation must then be replaced by one that is properly frame invariant and can therefore properly account for finite strains.

\section{APPENDIX}

Let equations (9), (10), and (11) be substituted into equation (15). This results in the following equation for $u(r, t)$,

$$
\begin{aligned}
G_{1}[\xi(r, t)] & D u(r, 0)+\int_{0}^{t} G_{1}[\xi(r, t)-\xi(r, s)] \frac{\partial}{\partial s}(D u(r, s)) d s \\
& +\int_{0-}^{t}\left\{\frac{\partial}{\partial r} G_{1}[\xi(r, t)-\xi(r, s)]\right\} \frac{\partial}{\partial s}\left(\frac{\partial u(r, s)}{\partial r}\right) d s \\
& +\int_{0-}^{t}\left\{\frac{\partial}{\partial r} G_{2}[\xi(r, t)-\xi(r, s)]\right\} \frac{\partial}{\partial s}\left(\frac{u(r, s)}{r}\right) d s=0,
\end{aligned}
$$

where $G_{1}[\xi(r, t)] \equiv K[\xi(r, t)]+\frac{4}{3} \mu[\xi(r, t)]$ and $G_{2}[\xi(r, t)] \equiv K[\xi(r, t)]-\frac{2}{3} \mu[\xi(r, t)] . D$ is the linear operator on the variable $r$ defined by

$$
D u(r, t) \equiv \frac{\partial}{\partial r}\left(\frac{\partial u(r, t)}{\partial r}\right)+\frac{1}{r} \frac{\partial u(r, t)}{\partial r}-\frac{u(r, t)}{r^{2}} .
$$

Taking the partial derivative of $G_{\alpha}, \alpha=1$ or 2 , with respect to $r$ gives

$$
\frac{\partial G_{\alpha}[\xi(r, t)-\xi(r, s)]}{\partial r}=\dot{G}_{\alpha}[\xi(r, t)-\xi(r, s)] \frac{\partial[\xi(r, t)-\xi(r, s)]}{\partial r}
$$


where $\dot{G}_{\alpha}$ denotes the derivative of $G_{\alpha}$ with respect to its argument. Recall the assumption that $f=f(\theta)$ in equation (7) and, hence, that $\xi(r, t)$ is now given by equation (17). Since $r$ and $t$ are independent variables,

$$
\frac{\partial[\xi(r, t)-\xi(r, s)]}{\partial r}=\int_{s}^{t} H[\theta(r, x)] \frac{\partial \theta(r, x)}{\partial r} d x
$$

with

$$
H[\theta(r, x)]=-\frac{1}{\phi^{2}(\theta(r, x))} \dot{\phi}(\theta(r, x)),
$$

and $\dot{\phi}$ denotes the derivative of $\phi$ with respect to its argument. It can be shown from equations (13) and (42) that

$$
\frac{\partial \theta(r, x)}{\partial r}=D u(r, x) .
$$

Combining equations (43), (44), and (46) gives

$$
\frac{\partial G_{\alpha}[\xi(r, t)-\xi(r, s)]}{\partial r}=\dot{G}_{\alpha}[\xi(r, t)-\xi(r, s)] \int_{s}^{t} H[\theta(r, x)] D u(r, x) d x .
$$

Expanding the terms in equation (41) as in equation (6), and using equation (47), the equilibrium equation becomes

$$
\begin{gathered}
G_{1}[\xi(r, t)] D u(r, 0)+\int_{0}^{t} G_{1}[\xi(r, t)-\xi(r, s)] \frac{\partial}{\partial s}(D u(r, s)) d s \\
+\frac{\partial u(r, 0)}{\partial r} \dot{G}_{1}[\xi(r, t)] \int_{0}^{t} H[\theta(r, x)] D u(r, x) d x \\
+\int_{0}^{t} \int_{s}^{t} \dot{G}_{1}[\xi(r, t)-\xi(r, s)] H[\theta(r, x)] D u(r, x) \frac{\partial}{\partial s}\left(\frac{\partial u(r, s)}{\partial r}\right) d x d s \\
+\dot{G}_{2}[\xi(r, t)]\left[\frac{u(r, 0)}{r}\right] \int_{0}^{t} H[\theta(r, x)] D u(r, x) d x \\
+\int_{0}^{t} \int_{s}^{t} \dot{G}_{2}[\xi(r, t)-\xi(r, s)] H[\theta(r, x)] D u(r, x) \frac{\partial}{\partial s}\left(\frac{u(r, s)}{r}\right) d x d s=0 .
\end{gathered}
$$

This is a partial differential - Volterra integral equation for $u(r, t)$. Note that each term in equation (48) contains $D u(r, x)$, for some set of values of the variable $x$. Thus, by inspection, a sufficient condition for equation (48) to be satisfied is that

$$
D u(r, t) \equiv \frac{\partial}{\partial r}\left(\frac{\partial u(r, t)}{\partial r}\right)+\frac{1}{r} \frac{\partial u(r, t)}{\partial r}-\frac{u(r, t)}{r^{2}}=0
$$

for $r_{i} \leq r \leq r_{0}, t \geq 0 . D u(r, 0)$ is a necessary and sufficient condition that equation (48) be met at $t=0$. It can be expected that $D u(r, t)=0$ is also a necessary and sufficient condition for some time interval beginning at $t=0$. Owing to the complexity of equation (48), no attempt is made here to determine whether this holds for all times. 
Acknowledgments. A. Wineman gratefully acknowledges the assistance of the Institute for Mechanics and Materials at the University of California, San Diego.

\section{REFERENCES}

[1] Shay, R. M., Jr. and Caruthers, J. M.: A new nonlinear viscoelastic constitutive equation for predicting yield in amorphous polymers. Journal of Rheology, 30, 781-827, (1986).

[2] Knauss, W. G. and Emri, I. J.: Non-linear viscoelasticity based on free volume consideration. Computers and Structures, 13, 123-128, (1981).

[3] Knauss, W. G. and Emri, I. J.: Volume change and the nonlinearly thermo-viscoelastic constitution of polymers. Polymer Engineering and Science, 27, 86-100, (1987).

[4] McKenna, G. B. and Zapas, L. J.: Nonlinear viscoelastic behavior of poly(methylmethacrylate) in torsion. Journal of Rheology, 23, 151-166, (1979).

[5] Moran, B. and Knauss, W.G.: Crack-tip stress and deformation fields in strain-softening nonlinearly viscoelastic materials. Journal of Applied Mechanics, 59, 95-101, (1992).

[6] Wineman, A. S. and Waldron, Jr., W. K.: Interaction of nonhomogeneous shear, nonlinear viscoelasticity and yield of a solid polymer. Polymer Engineering and Science, 33, 1217-1228, (1993).

[7] Wineman, A. S., and Kolberg, R. F.: Mechanical response of beams of a nonlinear viscoelastic material. Polymer Engineering and Science, 35, 345-350, (1995).

[8] Kolberg, R. F.: Application of a Nonlinear Viscoelastic Constitutive Equation to Isothermal Pure Bending near Material Yield Point, Ph.D. Thesis, The University of Michigan, Ann Arbor, 1994.

[9] Min, J.-H.: Dilatation Enhanced Stress Relaxation Effects in the Nonlinear Viscoelastic Solid Polymeric Structures, Ph.D. Thesis, The University of Michigan, Ann Arbor, 1995. 